\title{
Analysis of Damage and Failure in Anisotropic Ductile Metals Based on Biaxial Experiments with the $\mathrm{H}$-Specimen
}

\author{
M. Brünig ${ }^{1} \cdot$ S. Koirala ${ }^{1} \cdot$ S. Gerke ${ }^{1}$
}

Received: 29 June 2021 / Accepted: 31 August 2021 / Published online: 28 September 2021

(c) The Author(s) 2021

\begin{abstract}
Background Dependence of strength and failure behavior of anisotropic ductile metals on loading direction and on stress state has been indicated by many experiments. To realistically predict safety and lifetime of structures these effects must be taken into account in material models and numerical analysis.

Objective The influence of stress state and loading direction on damage and failure behavior of the anisotropic aluminum alloy EN AW-2017A is investigated.

Methods New biaxial experiments and numerical simulations have been performed with the H-specimen under different load ratios. Digital image correlation shows evolution of strain fields and scanning electron microscopy is used to visualize failure modes on fracture surfaces. Corresponding numerical studies predict stress states to explain damage and fracture processes on the micro-scale.

Results The stress state, the load ratio and the loading direction with respect to the principal axes of anisotropy affect the width and orientation of localized strain fields and the formation of damage mechanisms and fracture modes at the micro-level.

Conclusions The enhanced experimental program with biaxial tests considering different loading directions and load ratios is suggested for characterization of anisotropic metals.
\end{abstract}

Keywords Anisotropic ductile metals $\cdot$ Damage and failure $\cdot$ Biaxial experiments $\cdot$ Stress state dependence $\cdot$ Loading direction dependence

\section{Introduction}

During the last decades many high quality metals and alloys have been developed to fulfill demands from lightweight industry. The main requests are improvement of cost efficiency, reduction in energy consumption as well as increase of safety and lifetime [1,2]. In this context, due to their considerable lightness and quality various aluminum alloys are widely used in different engineering disciplines. However, during loading of structural elements often various stress-state-dependent failure mechanisms occur on different scales. For example, damage processes on the micro-scale can lead to ductile fracture on the macro-level and this can

M. Brünig

michael.bruenig@unibw.de

1 Universität der Bundeswehr München, Institut für Mechanik und Statik, Werner-Heisenberg-Weg 39, Neubiberg 85579, Germany cause the end of life of engineering structures. On the other hand, optimization of material properties led to reduction of localization of irreversible deformations as well as of these undesired damage and fracture events.

Uncertainties in manufactured parts may cause remarkable problems in automotive or aeronautical applications and, therefore, accurate prediction of their performances is very important. As a consequence, detailed analysis of irreversible deformations as well as damage and fracture behavior of these optimized metals and alloys is one of the main issues in engineering [3] allowing proposition of accurate and practically applicable constitutive models $[4,5]$. These theoretical frameworks and corresponding numerical programs must be based on experiments taking into account different loading directions with respect to the principal axes of anisotropy and a wide range of multi-axial loading conditions causing different stress states to be able to identify material parameters and to validate the constitutive theories for various engineering applications [6]. Focus of the 
new experimental-numerical investigation presented in this paper is on the effect of different stress states and of loading direction with respect to the principal axes of anisotropy on damage and failure behavior in anisotropic metals especially for shear-dominated loading conditions.

In the literature, analysis of evolution of inelastic deformations as well as damage and fracture processes on both the micro- and the macro-level is based on a series of experiments with different uni- and biaxially loaded specimens with different geometries. In particular, uniaxial tension tests with unnotched and differently notched specimens have been performed to analyze the influence of stress state on inelastic deformation behavior and on damage mechanisms [7-9] or to investigate fracture in ductile metals [10-13]. In addition, examination of shear-dominated stress states requires special geometries of specimens undergoing shear deformation which have been proposed in the literature [14-16]. On the other hand, an extended testing program with biaxially loaded cruciform specimens has been developed because the experiments with uniaxially loaded specimens can only cover a small band of stress triaxialities [17-20]. Special design and manufacturing of a mechanical device has been proposed to carry out biaxial experiments in a uniaxial testing machine [21]. Optimization of the geometries of the biaxially loaded cruciform specimens has been discussed to receive required stress states [22-25]. For example, a cruciform hole expansion test has been presented to investigate the quality of different yield functions [26]. In addition, alternative geometries of biaxially loaded cruciform specimens have been proposed to analyze the effect of different load paths and stress states on inelastic deformation as well as damage and fracture behavior in ductile metals [6, 27-29]. However, in these studies only isotropic metals have been examined.

In the manufacturing process aluminum alloys are deformed by various forming operations such as rolling, deep drawing or extrusion to deliver thin sheets for structural components, for example, in automotive and aeronautical industries. During these forming processes large inelastic deformations occur leading to anisotropies in the used ductile metals caused by internal changes in the crystallographic texture. Therefore, these deformation-induced anisotropies have to be taken into account in appropriate constitutive theories and corresponding numerical approaches to predict the mechanical behavior of ductile metal sheets in an accurate manner. In particular, Hill [30] developed a quadratic function of stresses which can be interpreted as an anisotropic generalization of the von Mises criterion. With the assumption of plastic incompressibility $r$-values based on measurement of plastic strain increments are defined to identify the plastic material parameters for thin sheets. A non-associated version of the criterion has been successfully used to develop an anisotropic damage model to predict failure of a high-strength pipeline steel [31]. However, in some applications the quadratic yield function was not able to accurately model both the yield stresses and the $r$-values and, therefore, alternative procedures to identify material parameters or enhanced yield criteria have been developed. For example, the virtual fields method has been applied to identify parameters of the anisotropic Hill condition from full-field measurement [32]. In addition, yield criteria using non-quadratic functions have been proposed to simulate anisotropic plastic behavior of metals [33-35]. Results of different uniaxial and equi-biaxial tension tests are used to identify the material parameters for these anisotropic yield criteria. An alternative quadratic yield function in combination with four hardening curves taken from uniaxial tension tests along rolling, diagonal and transverse directions as well as from equi-biaxial tests has been discussed [36]. Alternatively, a spline yield condition has been proposed [26] using results of the cruciform hole expansion test showing better accuracy than conventional anisotropic yield functions [30,33].

Ductile metal workpieces undergo various stress states during the metal forming processes causing damage mechanisms on the micro-level which can accumulate to unexpected fracture on the macro-scale. Thus, it is important to detect these failure processes and to understand the damage mechanisms on the micro-level as well as their transition to the macro-scale. To get more insight in this complex behavior of anisotropic ductile metals a new series of innovative experiments has been performed with biaxially loaded specimens taking into account different load ratios and various loading directions with respect to the principal axes of anisotropy. In the present paper, the behavior of the H-specimen under biaxial loading conditions leading to shear-dominated stress states is investigated in detail. The effect of loading conditions on localized inelastic strains as well as on damage and failure processes is examined using a combined experimental-numerical analysis. Material properties of the investigated aluminum alloy EN AW-2017A are determined by uniaxial tension tests with dog-bone-shaped specimens and by uniaxial shear tests with smile-shape specimens taken from metal sheets in different directions. The main aspect of the paper are new experiments with the biaxially loaded $\mathrm{H}$-specimen which have been carried out with focus on the influence of anisotropy-direction-dependent loading conditions on damage and fracture mechanisms on both the microand the macro-level. In these tests digital image correlation monitors evolution of strain fields in selected regions of the $\mathrm{H}$-specimen during the loading process. Scanning electron microscopy of the fractured surfaces elucidates different damage and fracture processes on the micro-level. Furthermore, results of corresponding numerical simulations detect stress states in critical parts of the specimens depending on loading directions and load ratios. These stress states are used to explain formation of the revealed damage and fracture processes. 


\section{Theory}

\section{Constitutive Model}

Modeling and numerical simulation of elastic-plastic deformation behavior of anisotropic ductile metals is based on experimental results on the macro-level. In the proposed phenomenological framework the strain rate tensor

$\dot{\epsilon}_{i j}=\dot{\epsilon}_{i j}^{e l}+\dot{\epsilon}_{i j}^{p l}$

is additively decomposed into an elastic $\dot{\epsilon}_{i j}^{e l}$ and a plastic part $\dot{\epsilon}_{i j}^{p l}$. For the investigated aluminum alloy the elastic behavior is taken to be isotropic. It is described by Hooke's law and, thus, the stress tensor is given by

$\sigma_{i j}=2 G \epsilon_{i j}^{e l}+\left(K-\frac{2}{3} G\right) \epsilon_{k k}^{e l} \delta_{i j}$

where $G$ and $K$ represent the shear and the bulk modulus, respectively, and $\delta_{i j}$ denotes the Kronecker delta (components of the unit tensor). In rolled sheets plastic anisotropy caused by the manufacturing process has to be taken into account. It is assumed that the axes of anisotropy coincide with the principal axes of the stresses. The principal axes of anisotropy lie in the $x$-direction of rolling $\left(\mathrm{RD}, 0^{\circ}\right)$, in the $y$-direction transversely in the plane of the sheet (TD, $90^{\circ}$ ) and in the $z$-direction normal to this plane. Onset of plastic yielding of anisotropic ductile metal is governed by Hill's [30] yield criterion

$$
\begin{array}{r}
f^{p l}\left(\sigma_{i j}\right)=\left[\frac { 1 } { 2 } \left\{F\left(\sigma_{y}-\sigma_{z}\right)^{2}+G\left(\sigma_{z}-\sigma_{x}\right)^{2}\right.\right. \\
+H\left(\sigma_{x}-\sigma_{y}\right)^{2}+2 L \sigma_{y z}^{2}+2 M \sigma_{x z}^{2} \\
\left.\left.+2 N \sigma_{x y}^{2}\right\}\right]^{1 / 2}-\bar{\sigma}=0
\end{array}
$$

where $F, G, H, L, M$ and $N$ are material parameters and $\bar{\sigma}$ represents the equivalent yield stress of a chosen reference test. These constitutive parameters are identified by different uniaxial tests.

In addition, formation of plastic strains during the loading process is modeled by an associated flow rule. It is assumed that the principal axes of the plastic strain rates coincide with those of the stresses. Then, the rate of the plastic strain tensor can be expressed in the form

$\dot{\epsilon}_{i j}^{p l}=\dot{\bar{\lambda}} \frac{\partial f^{p l}}{\partial \sigma_{i j}}$

where $\bar{\lambda}$ is a non-negative factor characterizing the amount of plastic strain rates. Based on the results of an experimental program with tension and compression tests of ductile metals superimposed by different hydrostatic pressures [37] plastic incompressibility $\left(\dot{\epsilon}_{i i}^{p l}=0\right)$ is taken into account.
In thin metal sheets identification of anisotropic material parameters is often restricted to experiments with specimens cut in its plane. Then, plane stress conditions $\left(\sigma_{z}=\sigma_{x z}=\sigma_{y z}=0\right)$ can be taken into account and the yield criterion (3) reduces to

$$
\begin{array}{r}
f_{s}^{p l}=\left[\frac { 1 } { 2 } \left\{(G+H) \sigma_{x}^{2}-2 H \sigma_{x} \sigma_{y}+(F+H) \sigma_{y}^{2}\right.\right. \\
\left.\left.+2 N \sigma_{x y}^{2}\right\}\right]^{1 / 2}-\bar{\sigma}=0 .
\end{array}
$$

The corresponding plastic strain rates (4) can be expressed in the reduced form

$\dot{\epsilon}_{x}^{p l}=\dot{\lambda}\left[(G+H) \sigma_{x}-H \sigma_{y}\right]$

$\dot{\epsilon}_{y}^{p l}=\dot{\lambda}\left[(F+H) \sigma_{y}-H \sigma_{x}\right]$

$\dot{\epsilon}_{x y}^{p l}=\dot{\lambda} N \sigma_{x y}$.

Based on the restriction of incompressible plastic deformations the rate of plastic strains in through-thickness direction is

$\dot{\epsilon}_{z}^{p l}=-\left(\dot{\epsilon}_{x}^{p l}+\dot{\epsilon}_{y}^{p l}\right)=-\dot{\lambda}\left(G \sigma_{x}+F \sigma_{y}\right)$.

Different uniaxial tension tests with specimens cut at an angle $\alpha$ to the rolling direction of the thin sheet are performed to determine the material parameters of the proposed elastic-plastic model. For example, the yield stresses in the principal directions of anisotropy can then be expressed in the form

$\sigma_{x}=\sigma_{\alpha} \cos ^{2} \alpha, \sigma_{y}=\sigma_{\alpha} \sin ^{2} \alpha, \sigma_{x y}=\sigma_{\alpha} \sin \alpha \cos \alpha$

where $\sigma_{\alpha}$ is the tensile yield stress in the $\alpha$-direction. With (equations (6) and (7)) the corresponding plastic strain rates are

$\dot{\epsilon}_{x}^{p l}=\dot{\lambda} \sigma_{\alpha}\left[(G+H) \cos ^{2} \alpha-H \sin ^{2} \alpha\right]$

$\dot{\epsilon}_{y}^{p l}=\dot{\lambda} \sigma_{\alpha}\left[(F+H) \sin ^{2} \alpha-H \cos ^{2} \alpha\right]$

$\dot{\epsilon}_{z}^{p l}=-\dot{\lambda} \sigma_{\alpha}\left(G \cos ^{2} \alpha+F \sin ^{2} \alpha\right)$

$\dot{\epsilon}_{x y}^{p l}=\dot{\lambda} \sigma_{\alpha} N \sin \alpha \cos \alpha$.

An alternative indirect method to determine the anisotropy parameters is based on the determination of ratios of measured plastic strain increments in tensile specimens cut in the $x$ - and $y$-direction of the thin sheet as well as in its diagonal direction (DD, $45^{\circ}$ ). Using the transformation of the plastic strain rates to the specimen's orientation $\alpha$ and the corresponding transverse direction $\left(\alpha+90^{\circ}\right)$ the respective strain rates are given by

$$
\begin{aligned}
\dot{\epsilon}_{\alpha}^{p l} & =\dot{\epsilon}_{x}^{p l} \cos ^{2} \alpha+\dot{\epsilon}_{y}^{p l} \sin ^{2} \alpha+2 \dot{\epsilon}_{x y}^{p l} \sin \alpha \cos \alpha \\
\dot{\epsilon}_{\alpha+90^{\circ}}^{p l} & =\dot{\epsilon}_{x}^{p l} \sin ^{2} \alpha+\dot{\epsilon}_{y}^{p l} \cos ^{2} \alpha-2 \dot{\epsilon}_{x y}^{p l} \sin \alpha \cos \alpha
\end{aligned}
$$


and with (equation (9)) the requested plastic strain rate can be expressed in the form

$\dot{\epsilon}_{\alpha+90^{\circ}}^{p l}=\dot{\lambda} \sigma_{\alpha}\left[-H+(G+F-2 N+4 H) \sin ^{2} \alpha \cos ^{2} \alpha\right]$.

The identification of the material parameters takes into account the definition of the $r$-values representing ratios of the plastic strain increments in different directions $\left(\alpha+90^{\circ}\right)$ and the through-thickness direction $z$. With (equation (7)) this leads to

$r_{\alpha}=\frac{\dot{\epsilon}_{\alpha+90^{\circ}}^{p l}}{\dot{\epsilon}_{z}^{p l}}=\frac{-\dot{\epsilon}_{\alpha+90^{\circ}}^{p l}}{\dot{\epsilon}_{x}^{p l}+\dot{\epsilon}_{y}^{p l}}$.

Taking into account (equations (9) and (11)) the $r$-value can be written in the form

$r_{\alpha}=\frac{H+(2 N-F-G-4 H) \sin ^{2} \alpha \cos ^{2} \alpha}{F \sin ^{2} \alpha+G \cos ^{2} \alpha}$

which is used to identify the respective anisotropy parameters of the yield condition (equation (5)).

\section{Material and Constitutive Parameters}

The investigated ductile material is the aluminum alloy EN AW-2017A (EN AW-AlCu4MgSi) supplied in the form of $4 \mathrm{~mm}$ thick sheets. Identification of material parameters is based on tensile and shear tests with specimens cut in different directions $\left(0^{\circ}, 45^{\circ}, 90^{\circ}\right)$ from the aluminum alloy sheets. For the tension tests, the geometry of the flat, dog-boneshaped specimen is shown in Fig. 1(a). In addition, shear tests have been performed with newly developed specimens with geometry shown in Fig. 1(b). The uniaxial experiments are performed in a uniaxial table test machine type inspekt table 50-1 (produced by Hegewald \& Peschke, Nossen, Germany) shown in Fig. 2. Digital image correlation is used to measure three-dimensional displacement fields on the respective surfaces of the specimens based on a special camera and lighting system schematically shown in Fig. 2(a). Four cameras are used to analyze the three-dimensional displacement. With a two camera stereo setting the out-of-plane movements can be measured and curved surfaces can be observed, while the central region of interest has to maintain within the depth of field during the series of reported pictures. Consequently, in the uniaxial tests four cameras are used (one pair on the front and one pair on the back surface) which have been calibrated by double sided calibration targets within a common coordinate system. This gives information of the 3D displacement fields on both surfaces including changes in the thickness. (a)

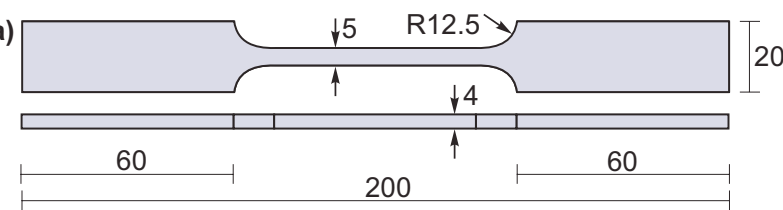

(b)

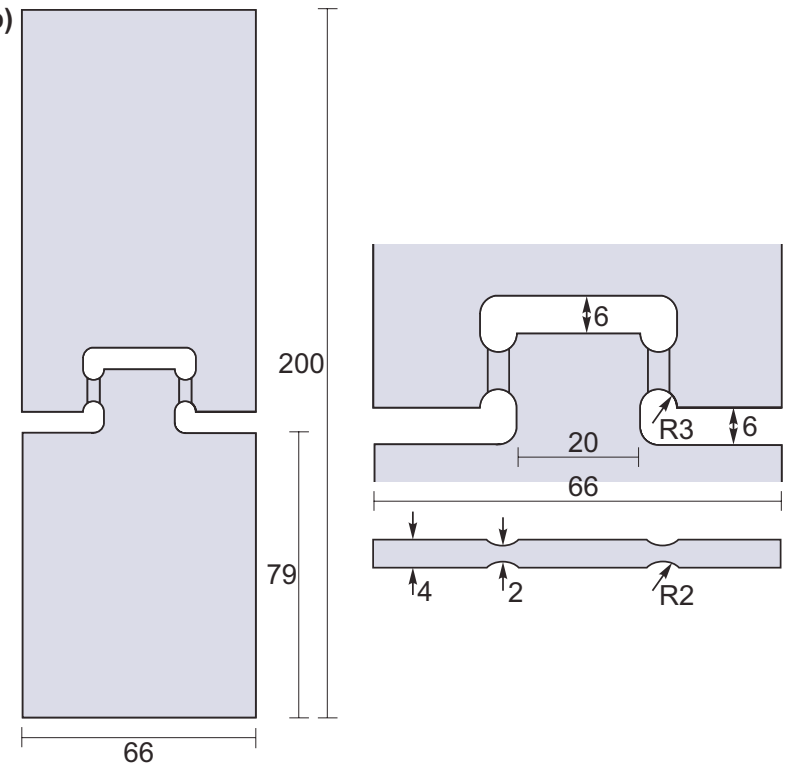

Fig. 1 Geometry of (a) the tensile specimen and (b) the shear specimen (all dimensions in $\mathrm{mm}$ )

Stress-strain curves of the uniaxial tests are analyzed. For example, isotropic elastic behavior is characterized by Young's modulus $E=74,000 \mathrm{MPa}$ and Poisson's ratio $v=0.3$. Description of the plastic behavior is based on the reference yield stress $\bar{\sigma}$ which is taken to be $\sigma_{0}$ of the tensile specimen (Fig. 1(a)) cut in rolling direction (RD). Plastic hardening of the investigated aluminum alloy EN AW 2017A is adequately described by the Voce law [38] for the respective current yield stresses

$c=c_{o}+R_{o} \epsilon^{p l}+R_{\infty}\left(1-e^{-b \epsilon^{p l}}\right)$

with the initial yield stress $c_{o}$, the hardening moduli $R_{o}$ and $R_{\infty}$, the hardening exponent $b$ as well as the equivalent

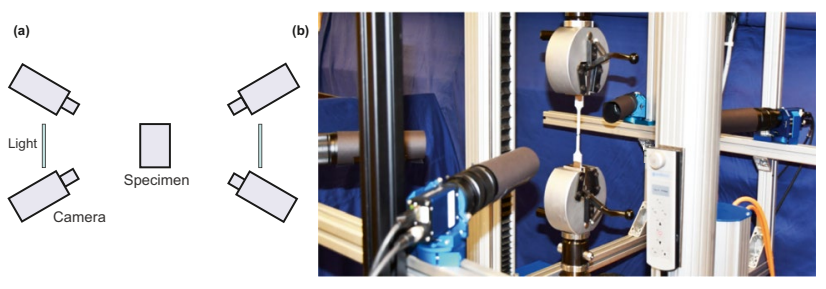

Fig. 2 (a) Schematic draw and (b) experimental setup for the uniaxial tests 
Table 1 Plastic material parameters

\begin{tabular}{lllll}
\hline & $c_{o}[\mathrm{MPa}]$ & $R_{o}[\mathrm{MPa}]$ & $R_{\infty}[\mathrm{MPa}]$ & $\mathrm{b}$ \\
\hline RD & 313 & 464 & 147 & 20 \\
DD & 308 & 474 & 127 & 28 \\
TD & 297 & 445 & 128 & 25 \\
Shear & 168 & 250 & 176 & 22 \\
\hline
\end{tabular}

plastic strain $\epsilon^{p l}$. For RD, DD and TD the respective material parameters are shown in Table 1. Comparison of experimentally obtained and numerically predicted stress-plastic strain curves taken from tension tests is shown in Fig. 3(a) with excellent agreement.

Furthermore, uniaxial tests with the shear specimen (Fig. 1(b)) have been carried out and results of the specimen loaded in RD are shown in Fig. 4. The first principal strain field in the notched region of the specimen is monitored by digital image correlation (Fig. 4(a)) and numerical simulation of these experiments detect the distribution of the shear stress $\sigma_{x y}$ in the cross section of the notch (Fig. 4(b)). In particular, Fig. 4(a) shows a localized first principal plastic strain band and shortly before fracture occurs it reaches $\epsilon_{1}=0.38$ which will be used as a reference value in the analysis of the biaxial tests with the H-specimen. In addition, the shear stress is homogeneously distributed in the cross section of the notch (Fig. 4(b)) only with gradients at the boundaries. At the onset of plastic yielding the shear stress reaches $\sigma_{x y}=168 \mathrm{MPa}$ in the plateau with maximum values for loading in RD and the corresponding shear stress-plastic shear strain curves for all investigated loading directions are shown in Fig. 3(b). Again, excellent agreement of experimental and numerically fitted curves can be seen.

Based on the measured strain fields on the surfaces of the tensile specimens different $r$-values are determined using (equation (12)) (see Table 2). With these Lankford coefficients in RD, DD and TD the anisotropy coefficients in the yield criterion (5) can be determined. Taking into account the results of the tension test with the specimen cut in RD
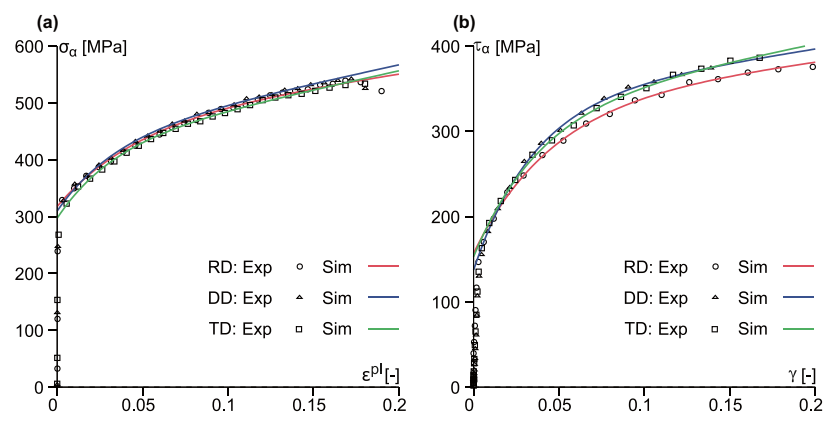

Fig. 3 Stress-plastic strain curves based on (a) the uniaxial tension test and (b) the shear test

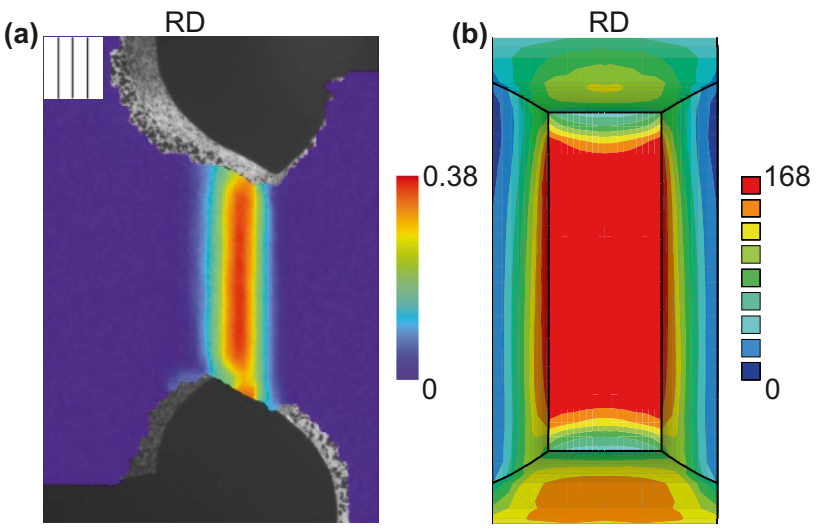

Fig. 4 Shear specimen: (a) first principal strain field and (b) shear stress $[\mathrm{MPa}]$

the yield condition (5) with $\sigma_{x}=\bar{\sigma}$ and (equation (13)) for $\alpha=0^{\circ}$ are used to compute the parameters

$G=\frac{2}{1+r_{0}}$

and

$H=2-G$.

In addition, with the results of the tension test with the specimen cut in TD the yield condition (5) leads to

$F=\frac{2 \bar{\sigma}^{2}}{\sigma_{y}^{2}}-H$

which can be seen as the stress-based definition of the parameter $F$. Alternatively, based on this experiment the anisotropy parameter $F$ can be computed using (equation (13)) for $\alpha=90^{\circ}$ :

$F=\frac{H}{r_{90}}$

which is the plastic strain-based value of the parameter $F$. Furthermore, with the results of the tension test with the specimen cut in DD (equation (13)) leads to the parameter

$N=\left(r_{45^{\circ}}+\frac{1}{2}\right)(F+G)$

which can be seen as the plastic strain-based value of the parameter $N$ taking into account the plastic strain increments occurring in the tension test with the specimen loaded in DD. Alternatively, taking into account the yield criterion (5) the

Table 2 Lankford coefficients

\begin{tabular}{lll}
\hline$r_{0}$ & $r_{45}$ & $r_{90}$ \\
\hline 0.597 & 0.783 & 0.695 \\
\hline
\end{tabular}


parameter $N$ can be directly identified by the shear test and, thus, is not based on the results of different tension tests.

Based on (equations (15), (16) and (18)) the parameters can be determined which are able to accurately predict the experimental $r$-values but leads to differences of numerically predicted yield stresses with experimental data. Thus, the parameter $F$ is alternatively identified by (equation (17)). However, this leads to deviations of the numerically predicted $r$-values compared with the experimental ones whereas the different yield stresses are well simulated. Therefore, the mean value of the respective parameters $F$ based on (equations (17) and (18)) is computed leading to acceptable numerical results for both the $r$-values and the yield stresses. In addition, in the present analysis the parameter $N$ is determined by the yield criterion (5) based on the shear test. It should be noted that based on (equation (19)) and the mean value of Parameter $F$ (based on (equations (17 and 18))) the parameter $N=3.2421$ is determined which is based on kinematic observations and assumptions. All anisotropy parameters are listed in Table 3 which are used in the various numerical simulations of the different loading processes of the $\mathrm{H}$-specimens.

\section{Experimental Procedure}

The uniaxial tests discussed above are used to identify the parameters of the elastic-plastic model and the corresponding numerical simulations. In addition, new biaxial experiments are performed to detect stress-state-dependent damage and fracture behavior in ductile materials as well as to elucidate the influence of loading direction in anisotropic metals. These experiments are carried out in the test machine type LFM-BIAX 20kN (produced by Walter \& Bai, Löhningen, Switzerland) containing four electro-mechanically driven actuators (Fig. 5(a)) allowing individual tensile or compressive loading with load maxima of $\pm 20 \mathrm{kN}$ in two orthogonal axes. Each cylinder is equipped with load cells of type Interface $1730 \mathrm{EGX}-20 \mathrm{kN}$ in standard flange design. Before the tests start the specimens are clamped in the four heads of the actuators. During the loading process the machine reports the displacements and the forces of each cylinder. The main demand of the biaxial testing technique is that the ratio of the forces $F_{1} / F_{2}$ in the respective axes is kept constant during loading leading to only marginal changes in the specific stress parameters (e.g. the stress triaxiality and the Lode parameter) in this case. In particular, the machine displacement $u_{1.1}^{M}$ of cylinder 1.1 in axis 1 (Fig. 6(e)) is continuously increased by $\frac{0.004 \mathrm{~mm}}{\mathrm{~s}}$. The same displacement is applied on the cylinder 1.2

Table 3 Anisotropy parameters

\begin{tabular}{llllll}
\hline$F$ & $G$ & $H$ & $L$ & $M$ & $N$ \\
\hline 1.2747 & 1.2523 & 0.7477 & 3. & 3. & 3.4711 \\
\hline
\end{tabular}
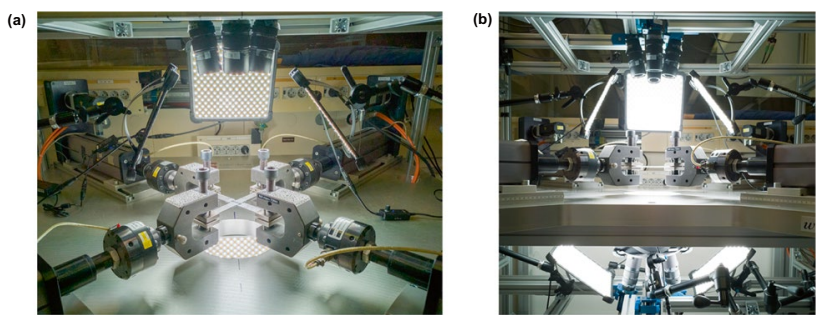

Fig. 5 (a) Biaxial test machine, (b) lighting system and camera equipment [39]

on the opposite side of the axis 1 as $u_{1.2}^{M}$. In the perpendicular axis 2 the cylinder 2.1 is force driven. This procedure guaranties constant load ratios during the loading process. The force in this cylinder leads here to the machine displacement $u_{2.1}^{M}$ and the same displacement is applied as $u_{2.2}^{M}$ on the cylinder 2.2 on the opposite side of the axis 2 . This experimental technique has been shown to be remarkably stable and allows performance of biaxial tests with constant load ratios causing nearly constant stress states during the proportional loading process. Consideration of different selected load ratios acting on the biaxially loaded cruciform specimens covers a wide range of requested multi-axial stress states.

Three-dimensional displacement fields in selected parts of the cruciform specimens are monitored during the loading process by Q-400 digital 3D image correlation (DIC) system provided by Dantec/Limess. Here, eight Allied Vision Manta G-609B/C cameras with sensors 2752 x 2206 Px; Sony IT CCD ICX694ALG/AQG equipped with Techspec 75mm DG Series, f/4-f/30, C-Mount lenses are used (Fig. 5(b)). The evaluation was realized with the corresponding Istra $4 \mathrm{D}$ software. The lighting system Fomex FL-B50 and Fomex FL-B25 (Fig. 5) is installed in such a way that shadows and reflections are avoided within the notched parts. Shortly before onset of the experimental loading process selected regions of the specimens were firstly sprayed with a white acrylic lacquer and, afterwards, the speckle pattern was sprayed on with a black, graphite-based coating. The pixel size, namely the relation between physical and sensor dimensions, is averaged $\frac{56 P x}{m m}$ and the DIC evaluation has been realized with a subset size
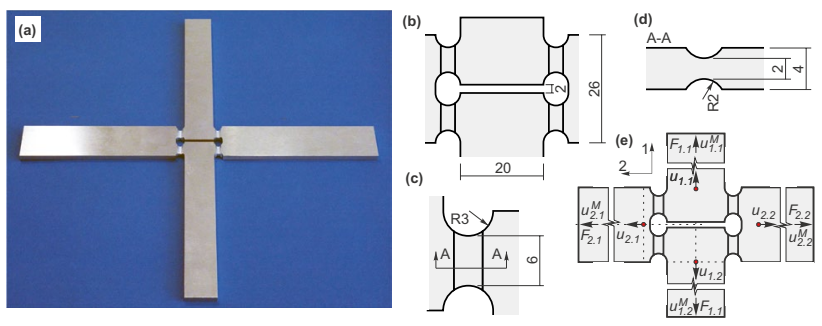

Fig. 6 Geometry, forces and displacements of the H-specimen (all dimensions in $\mathrm{mm}$ ) [39] 
of $33 P x$ with an overlap of $11 P x$ in both directions leading to 2.5 evaluation points per $\mathrm{mm}$. During the tests the displacements and the forces of the actuators are transferred and stored with the data sets of the digital image correlation system at a frequency of $1.0 \mathrm{~Hz}$. With this visualization technique evolution of strain fields on the boundaries of the specimens can be examined in detail and strain localization phenomena as well as onset of macro-cracking can be revealed. Furthermore, at the end of the tests fracture surfaces of the failed specimens are analyzed after the loading process by scanning electron microscopy (SEM) to visualize damage and fracture mechanisms on the micro-scale.

For experimental analysis of formation of inelastic deformation behavior and stress-state-dependent damage and fracture processes of ductile metals new geometries of cruciform specimens have been proposed [6]. In the present paper, the H-specimen (Fig. 6(a)) is used to study the influence of anisotropic material characteristics on multi-axial mechanical behavior of the investigated aluminum alloy sheets. During biaxial loading of the $\mathrm{H}$-specimen different combinations of shear and tension/compression stresses will occur in the notches allowing studies on the effect of sheardominated stress states on damage and failure of ductile metals. The outer dimensions of the $\mathrm{H}$-specimen are $240 \mathrm{~mm}$ in both directions. In its central part four notches in thickness direction are milled (b), leading here to localization of strain fields during the biaxial loading process. The length of each notched part is $6 \mathrm{~mm}$ and the reduction of thickness is up to $2 \mathrm{~mm}$. The notch radii are $3 \mathrm{~mm}$ in plane (c) and $2 \mathrm{~mm}$ in thickness (d) direction. The $\mathrm{H}$-specimen is simultaneously loaded in two perpendicular directions by the forces $F_{1}$ and $F_{2}$ (e) with different proportional load paths. Since the displacements of the actuators $u^{M}$ (Fig. 6(e)) include movements caused by machine stiffness and possible slipping at the clamping device they are not used to compare experimental and numerical results. Hence, displacements of the red points shown in Fig. 6(e), $u_{1.1}$ and $u_{1.2}$ in direction 1 and $u_{2.1}$ and $u_{2.2}$ in direction 2 , are recorded by DIC during the tests. They are used to determine the relative displacements $\Delta u_{\text {ref. } 1}=u_{1.1}-u_{1.2}$ and $\Delta u_{\text {ref. } 2}=u_{2.1}-u_{2.2}$ shown in the load-displacement curves.

Furthermore, numerical simulations of the respective biaxial experiments with the $\mathrm{H}$-specimen have been carried out to detect the stress states during the loading processes. For the numerical analysis the finite element program ANSYS has been used enhanced by a user-defined material subroutine based on the elastic-plastic theory discussed above. In particular, a quarter of the $\mathrm{H}$-specimen is divided into 20,802 eight-node-elements of type SOLID185 (Fig. 7) using symmetry boundary conditions. The respective displacements are applied to the nodes at the end faces and outof-plane movements are prevented by zero displacements of the nodes in the symmetry planes in out-of-plane direction.
Refinement of the finite element mesh is taken into account in the central parts of the $\mathrm{H}$-specimen to accurately predict the expected stress gradients and localization of strains.

\section{Results and Discussion}

In the present experimental program the $\mathrm{H}$-specimen is uniand biaxially loaded with four load ratios leading to different stress states in the notched regions. In particular, in the first case the $\mathrm{H}$-specimen is uniaxially loaded by $F_{1} / F_{2}=1 / 0$ leading to shear behavior in the notches. In all load cases, direction 1 (Fig. 6(e)) corresponds to the rolling direction (RD), the diagonal direction (DD) and the transverse direction (TD), respectively. In the second case, the specimen is uniaxially loaded by $F_{1} / F_{2}=0 / 1$ leading to tensile behavior in the notches. These uniaxial load ratios can be seen as reference loading scenarios to be compared with the biaxial ones. Then in the third case, the $\mathrm{H}$-specimen is biaxially loaded by $F_{1} / F_{2}=1 /+1$ leading to shear with superimposed tensile behavior in the notched parts. And in the fourth case, the specimen is biaxially loaded by $F_{1} / F_{2}=1 /-1$ where in the notches shear with superimposed compressive behavior is expected to occur.

Figure 8 shows experimental and numerically predicted load-displacement curves. In particular, in the experiments with the load ratio $F_{1} / F_{2}=1 / 0$ (Fig. $8(\mathrm{a})$ ) an increase in load is observed up to $F_{1}=7.5 \mathrm{kN}$ for loading in RD and the $\mathrm{H}$-specimen failed at the displacement $\Delta u_{\text {ref }}=1.79 \mathrm{~mm}$. For loading in DD the maximum load reaches $F_{1}=7.3 \mathrm{kN}$ whereas the final displacement at onset of fracture is $\Delta u_{\text {ref }}=$ $1.89 \mathrm{~mm}$. For loading in TD, the load increases up to $F_{1}=$ $7.5 \mathrm{kN}$ and failed at the displacement $\Delta u_{\text {ref }}=1.77 \mathrm{~mm}$. It can

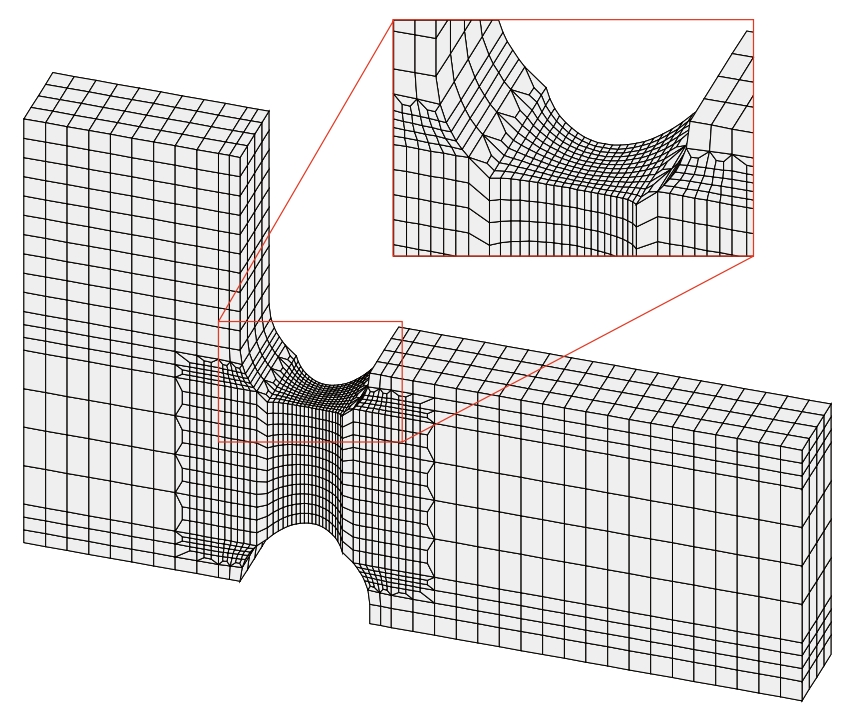

Fig. 7 Finite element mesh 
be clearly seen from the experiments with the H-specimen loaded by $F_{1} / F_{2}=1 / 0$ that the loading direction with respect to the principal directions of anisotropy has an influence on the maximum loads as well as on the displacements at failure. The loads differ about 3\% where loading in RD and TD leads to the highest loads whereas the smallest one is reached for loading in DD. The displacements at failure differ about $7 \%$ where the largest displacement is measured for loading in DD and the smallest one for loading in TD. Therefore, these experimental results show that for loading in RD the behavior is a little bit more brittle whereas loading in DD leads to slightly more ductile behavior.

In addition, numerical simulation of the tests with the $\mathrm{H}$-specimen has been carried out based on the elastic-plastic constitutive model discussed above and the material parameters obtained from uniaxial tension and shear tests. The numerical analysis is able to reproduce the experimental behavior and only small differences in the load maxima can be seen whereas the main trends are well predicted. These differences seem to be caused by the elastic-plastic analysis whereas in the experiments damage and failure processes take place leading to decrease in loads especially in the later stage of the tests with ongoing plastic deformation.

In the experiments with the load ratio $F_{1} / F_{2}=0 / 1$ (Fig. 8(b)) an increase in load is observed up to $F_{2}=12.8 \mathrm{kN}$ for orientation of the 1-axis in $\mathrm{RD}$ and the $\mathrm{H}$-specimen failed at the displacement $\Delta u_{\text {ref }}=0.51 \mathrm{~mm}$. For orientation in DD the maximum load reaches $F_{2}=12.4 \mathrm{kN}$ whereas the final displacement at onset of fracture is $\Delta u_{\text {ref }}=0.56 \mathrm{~mm}$. For orientation in TD, the load also increases up to $F_{2}=12.4 \mathrm{kN}$ and failed at the displacement $\Delta u_{\text {ref }}=0.57 \mathrm{~mm}$. Again, it can be seen from these experiments with the $\mathrm{H}$-specimen loaded by $F_{1} / F_{2}=0 / 1$ that the orientation of the 1 -axis with respect
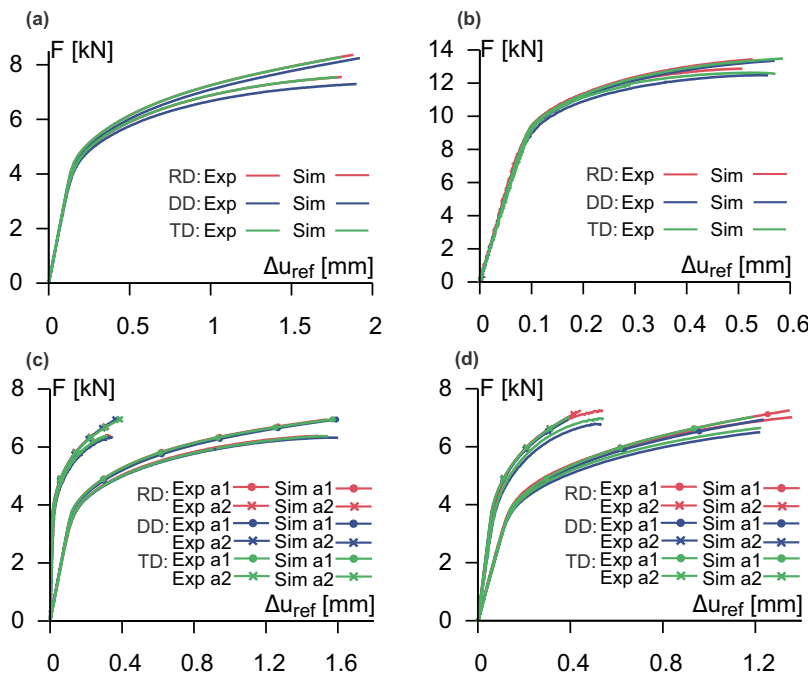

Fig. 8 Load-displacement curves for the load ratios (a) $F_{1} / F_{2}=1 / 0$, (b) $F_{1} / F_{2}=0 / 1$, (c) $F_{1} / F_{2}=1 /+1$, and (d) $F_{1} / F_{2}=1 /-1$ to the principal directions of anisotropy has an effect on the maximum loads as well as on the displacements at onset of fracture. The loads differ about $3 \%$ and orientation in RD leads to the highest load whereas the smallest ones are reached for orientation in DD and TD. The displacements at failure differ about $12 \%$ where the largest displacement is measured for orientation in TD and the smallest one for orientation in RD. Therefore, these experimental results show that for orientation in RD the behavior is more brittle whereas orientation in DD and TD leads to more ductile behavior. Corresponding numerical simulations have been performed showing good agreement with the experimental load-displacement curves. Only a small over-prediction in loads can be seen for this load ratio but the main trends are well reflected.

In the experiments with the load ratio $F_{1} / F_{2}=1 /+1$ (Fig. 8(c)) similar load-displacement curves are obtained. In all cases of the orientation of $F_{1}$-loading (RD, DD and TD) the load maxima are nearly identical with $F_{1}=F_{2}=$ $6.35 \mathrm{kN}$. However, the displacements at onset of fracture are different. For $F_{1}$-loading in RD the $\mathrm{H}$-specimen failed at $\Delta u_{\text {ref. } 1}=1.49 \mathrm{~mm}$ and $\Delta u_{\text {ref. } 2}=0.34 \mathrm{~mm}$. For $F_{1}$-loading in DD the displacements at fracture are $\Delta u_{\text {ref. } 1}=1.60 \mathrm{~mm}$ and $\Delta u_{\text {ref. } 2}=0.34 \mathrm{~mm}$. And for $F_{1}$-loading in TD the specimen failed at $\Delta u_{\text {ref. } 1}=1.54 \mathrm{~mm}$ and $\Delta u_{\text {ref. } 2}=0.34 \mathrm{~mm}$. The $F_{1}$ -loading direction with respect to the principal directions of anisotropy does not affect the load maxima but has an influence on the displacements at onset of fracture and the differences are about 7\%. Thus, the behavior in RD is more brittle whereas in DD it is more ductile. In addition, numerically predicted load-displacement curves are also shown in Fig. 8(c) simulating well the experimental behavior. Only an over-prediction of the loads can be seen but the main trends with nearly identical loads are nicely reproduced.

In the experiments with the load ratio $F_{1} / F_{2}=1 /-1$ (Fig. 8(d)) load-displacement curves for the positive and the negative load paths are obtained where $-F_{2}$ and $-\Delta u_{\text {ref. } 2}$ are shown in the diagram. For $F_{1}$-loading in $\mathrm{RD}$ the maximum loads are $F_{1}=7.06 \mathrm{kN}$ and $F_{2}=-7.24 \mathrm{kN}$ showing slightly non-equal behavior caused by inaccuracies in the experimental procedure during compressive loading. These difficulties can also be seen in the slight scattering of the $F_{2}-\Delta u_{\text {ref.2 }}$ -curves in the last load steps. In this case the $\mathrm{H}$-specimen failed at $\Delta u_{\text {ref } 1}=1.36 \mathrm{~mm}$ and $\Delta u_{\text {ref } 2}=-0.55 \mathrm{~mm}$. For $F_{1}$ -loading in DD and TD the loads increase up to $F_{1}=6.53 \mathrm{kN}$ and $F_{2}=-6.82 \mathrm{kN}$ whereas the displacements at onset of fracture $\Delta u_{\text {ref. } 1}=1.23 \mathrm{~mm}$ and $\Delta u_{\text {ref. } 2}=-0.55 \mathrm{~mm}$ have been measured for both cases. Thus, from these experiments with the $\mathrm{H}$-specimen loaded by $F_{1} / F_{2}=1 /-1$ it can be clearly seen that the $F_{1}$-loading direction with respect to the principal directions of anisotropy affects the load maxima and the displacements at the onset of fracture which differ about $8 \%$ and $11 \%$, respectively. They show that the behavior for $F_{1}$-loading in RD is more ductile compared to the 
other loading directions. In addition, numerical simulations of these experiments have been carried out. The numerical load-displacement curves are nearly identical for RD and TD whereas slightly smaller loads are numerically predicted for DD. The numerical analysis only slightly over-predicts the experimentally measured loads.

Strain fields in the notched regions are monitored by digital image correlation (DIC) technique during loading of the $\mathrm{H}$-specimen. Figure 9 shows first principal strain fields shortly before the onset of fracture occurs for different load ratios and for different orientations with respect to the principal directions of anisotropy. On the top of each figure the rolling direction is shown by the black lines. In particular, for the load ratio $F_{1} / F_{2}=1 / 0$ (Fig. 9(a)) with loading in RD small first principal strains bands can be seen with slightly diagonal orientation from top left to bottom right for the left upper notch. During the loading process the behavior in all four notches is nearly identical and shortly before failure happens faster increase in strains is observed in the lower notches with maxima $\epsilon_{1}=0.40$. It should be noted that this localized strain behavior has also been observed in the uniaxial shear specimens (Fig. 4(b)). Similar behavior is observed in the experiments with loading in DD but smaller strain maxima develop up to $\epsilon_{1}=0.35$. For loading in TD again
RD

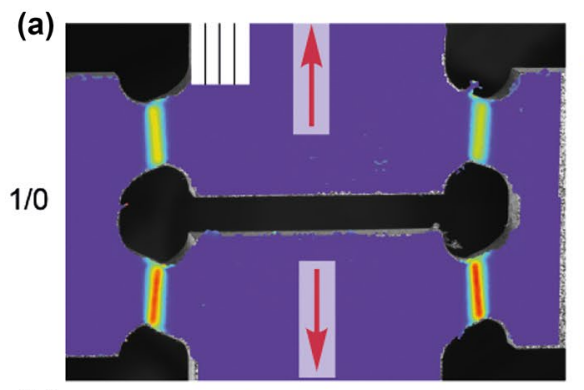

(b)

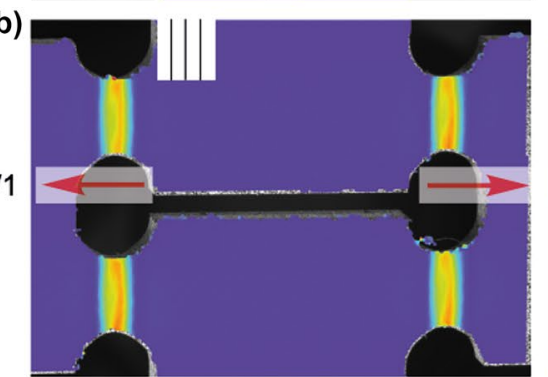

(c)

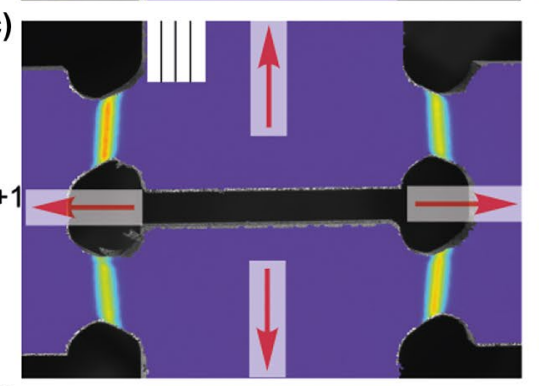

(d)

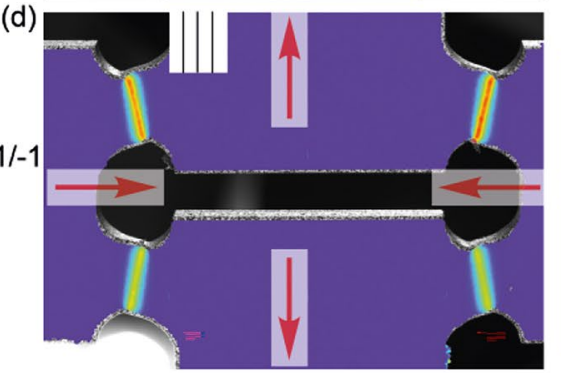

DD
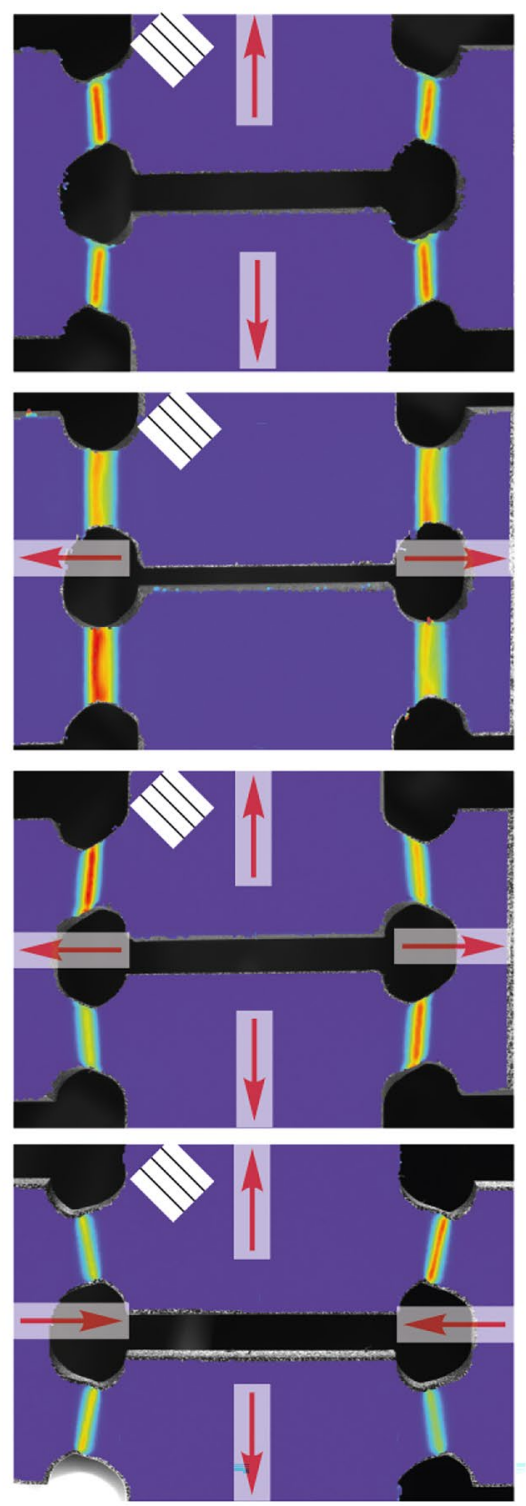

TD
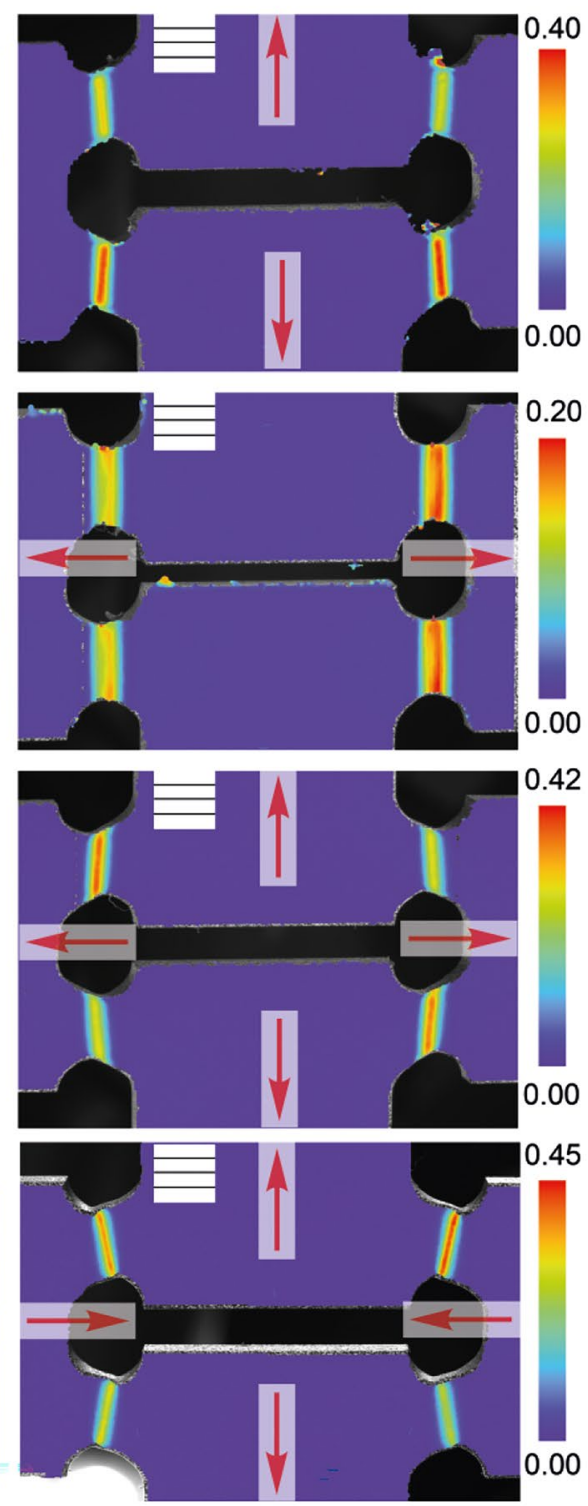

Fig. 9 First principal strain fields before fracture occurs for the load ratios (a) $F_{1} / F_{2}=1 / 0,(\mathbf{b}) F_{1} / F_{2}=0 / 1,(\mathbf{c}) F_{1} / F_{2}=1 /+1$, and (d) $F_{1} / F_{2}=1 /-1$ 
small principal strain bands occur with strain maxima up to $\epsilon_{1}=0.40$ in the lower notches. In summary, for $F_{1} / F_{2}=1 / 0$ small principal strain bands with the width of about $2 \mathrm{~mm}$ are formed with slightly diagonal orientation of $4^{\circ}$ with respect to the vertical axis and larger values for loading in RD and TD.

The experimental results for the load ratio $F_{1} / F_{2}=0 / 1$ are shown in Fig. 9(b). For the orientation of the 1-axis of the H-specimen in RD more widespread vertical bands of principal strains occur with maxima in an elliptical shape. The maxima are $\epsilon_{1}=0.15$ in the notches. Also wide vertical bands of principal strains are measured for specimen's orientation in DD with higher values up to $\epsilon_{1}=0.20$ in the left lower notch. And for orientation in TD again these widespread vertical bands can be seen with strain maxima up to $\epsilon_{1}=0.18$ in the right notches. In summary, for the load ratio $F_{1} / F_{2}=0 / 1$ for all orientations of the H-specimen more widespread bands with the width of about $3 \mathrm{~mm}$ occur and large principal strains develop with vertical orientation and the maxima form an ellipse.

For the load ratio $F_{1} / F_{2}=1 /+1$ (Fig. 9(c)) the principal strains are localized in small strain bands with the width of about $2 \mathrm{~mm}$ and with diagonal orientation of $6^{\circ}$ from bottom left to top right for the left upper notch. For $F_{1}$-loading in RD the maxima are $\epsilon_{1}=0.35$ whereas $\epsilon_{1}=0.42$ occurs for DD. In the case of $F_{1}$-loading in TD $\epsilon_{1}=0.38$ is reached shortly before failure happens.

Figure 9(d) shows the principal strains for the load ratio $F_{1} / F_{2}=1 /-1$. They are localized in very small strain bands with the width of about $2 \mathrm{~mm}$ and with diagonal orientation of $14^{\circ}$ from top left to bottom right for the left upper notch. For $F_{1}$-loading in RD the maxima are $\epsilon_{1}=0.45$ whereas $\epsilon_{1}=0.43$ is reached for DD. In the case of $F_{1}$-loading in TD $\epsilon_{1}=0.45$ is measured shortly before failure happens. In summary, distribution, localization and orientation of principal strain bands are mainly caused by the load ratio whereas the orientation of the $\mathrm{H}$-specimen during loading (RD, DD, or TD) affects the maximum values.

In Fig. 10 photos of the fractured specimens are shown for different load ratios and different orientations of the $\mathrm{H}$-specimen. In all cases, the directions of the fracture lines correspond to the localized first principal strain bands (Fig. 9) and remarkable inelastic deformations are also visible in the non-fractured notches indicating large strain behavior. In particular, for the load ratio $F_{1} / F_{2}=1 / 0$ (Fig. 10(a)) the fracture occurs in the lower notches and the fracture lines show slightly diagonal orientation from bottom left to top right in the left notch with shear-dominated fracture mode. In the case of the load ratio $F_{1} / F_{2}=0 / 1$ (Fig. 10(b)) vertical fracture lines can be seen with typical tensile-dominated fracture modes and cup-cone behavior. For the load ratio $F_{1} / F_{2}=1 /+1$ (Fig. 10(c)) the fracture lines are diagonally oriented from bottom left to top right in the left upper notch. For the load ratio $F_{1} / F_{2}=1 /-1$
(Fig. 10(d)) the fracture lines show diagonal orientation from bottom left to top right in the right upper notch. In this case, the fracture modes are typical shear-dominated ones with smooth fracture surfaces. These experimental results clearly show that fracture lines on the macroscopic level are affected by the load ratios whereas the influence of the loading direction (RD, DD or TD) is marginal.

Based on elastic-plastic numerical simulations of the respective experiments the stress states in the notched parts of the tested H-specimens can be detected. For example, the stress triaxialities $\eta$ (ratio of the mean stress and the equivalent stress) on the surface (S) and in the cross section (C) of the notches are shown in Fig. 11. For the load ratio $F_{1} / F_{2}=1 / 0$ (Fig. 11(a)) the distribution of the stress triaxiality in the cross section $(\mathrm{C})$ is nearly homogeneous with small gradients on the bottom and on the top. In the center of the notch the stress triaxiality $\eta=0.10$ is numerically predicted whereas on the top and on the bottom of the cross section $\eta=0.35$ is reached. The effect of the orientation of the specimen (RD, DD, or TD) on the stress triaxialities for this loading case is marginal. On the surface (S) of the notch the stress triaxiality shows a widespread diagonal band with $\eta=0.00$ whereas in the top left and bottom right corner $\eta=$ 0.50 and in the top right and bottom left corner $\eta=-0.70$ can be seen. For the load ratio $F_{1} / F_{2}=0 / 1$ (Fig. 11(b)) higher gradients of the stress triaxiality can be seen in the cross section (C) of the notched part of the $\mathrm{H}$-specimen. In the center of the notch the stress triaxiality $\eta=1.00$ is reached whereas on the boundaries of the cross section (C) and on the surface (S) it is only $\eta=0.40$. For this loading case, the stress triaxialities for RD and TD are nearly identical whereas slightly smaller triaxialities are predicted in the area of the cross section (C) for DD. On the surface (S) of the notch the stress triaxiality is also nearly homogeneous with $\eta=0.40$. For the load ratio $F_{1} / F_{2}=1 /+1$ the stress triaxialities are shown in Fig. 11(c). In this case the distribution of the stress triaxialiy in the cross section (C) of the notch is nearly homogeneous with only small gradients on the left and on the right side. In the center of the notch $\eta=0.50$ is numerically predicted whereas in small regions on the sides $\eta=0.20$ is reached. On the surface (S) of the notch the stress triaxiality shows a vertical band with $\eta=0.20$. For the load ratio $F_{1} / F_{2}=1 /-1$ the stress triaxialities are shown in Fig. 11(d). In this case the distribution of the stress triaxialiy in the cross section (C) of the notch is nearly homogeneous with only very small gradients on the bottom and on the top. In the center of the notch $\eta=-0.20$ is numerically predicted whereas in small regions on the top and on the bottom $\eta=$ 0.33 is reached. On the surface $(\mathrm{S})$ of the notch the stress triaxiality is also nearly homogeneous with $\eta=-0.20$ and only in the corners small gradients occur. Again, the influence of the specimen's orientation is marginal. These numerical results have clearly shown that the amount and distribution 

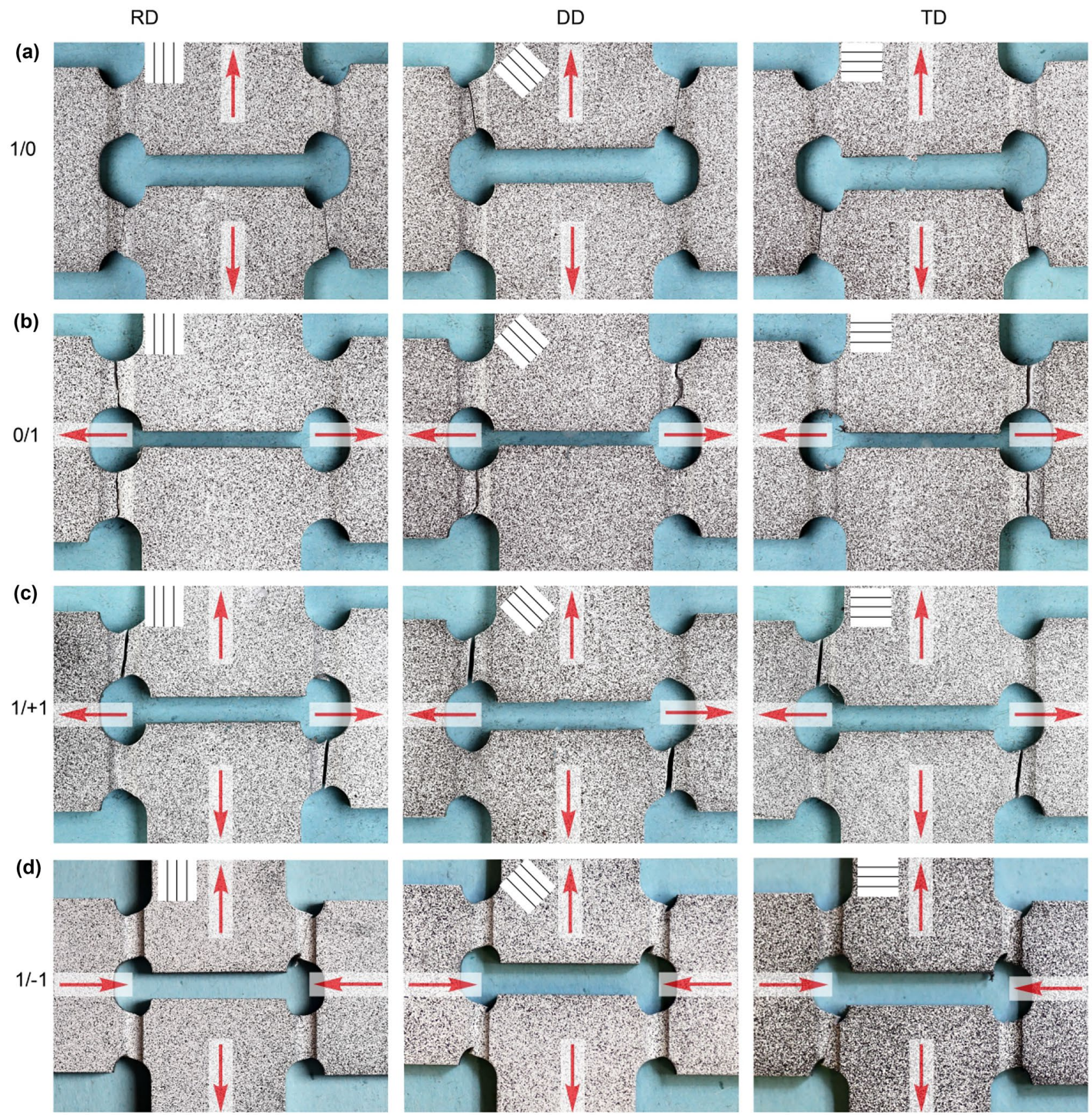

Fig. 10 Fractured specimens for the load ratios (a) $F_{1} / F_{2}=1 / 0$, (b) $F_{1} / F_{2}=0 / 1$, (c) $F_{1} / F_{2}=1 /+1$, and (d) $F_{1} / F_{2}=1 /-1$

of the stress triaxialities in the notches of the H-specimen remarkably depend on the load ratio whereas they are only marginally affected by the orientation of the specimen (RD, DD and TD).

After the tests the fracture surfaces have been examined by scanning electron microscopy (SEM). Figure 12 shows the respective pictures taken from pieces in the center of the fracture surfaces. For example, for the load ratio $F_{1} / F_{2}=1 / 0$ (Fig. 12(a)) small voids and shear mechanisms can be seen after loading in RD leading to shear cracks. For loading in DD micro-shear-cracks occur in combination with few small, sheared voids. For loading in TD again only very few voids are visible but remarkable micro-shear-cracks can be seen. However, for all loading directions the stress triaxiality was nearly identical with $\eta=0.00$ in the center of the notch (Fig. 11(a)). For this stress state in isotropic materials the shear failure mode with simultaneous shearing of small initial voids and formation of micro-shear-cracks is expected 


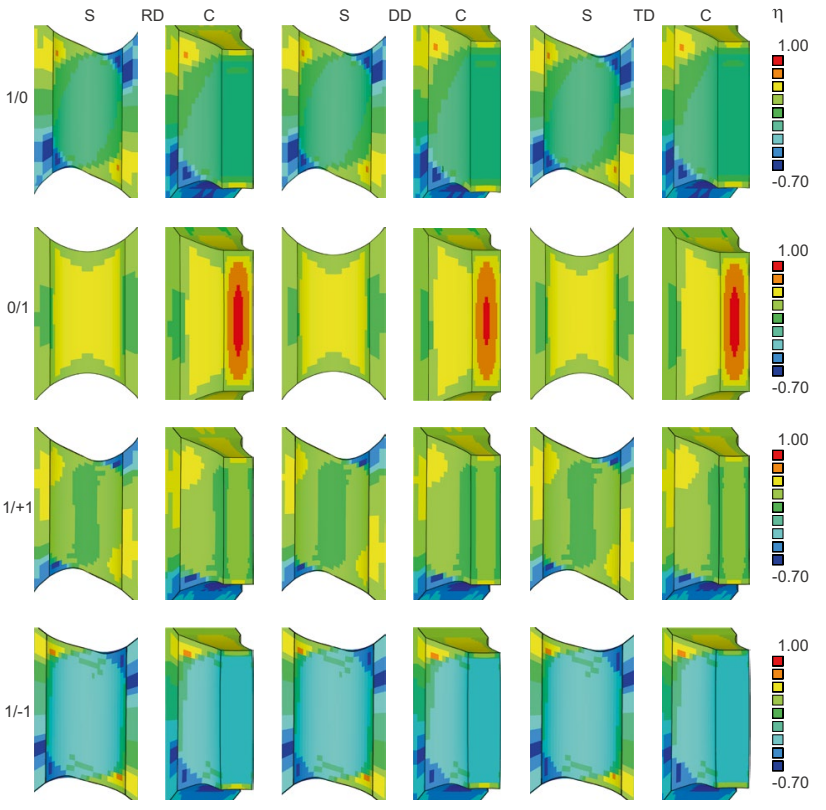

Fig. 11 Stress triaxialities $\eta$ for the load ratios (a) $F_{1} / F_{2}=1 / 0$, (b) $F_{1} / F_{2}=0 / 1$, (c) $F_{1} / F_{2}=1 /+1$, and (d) $F_{1} / F_{2}=1 /-1$

to occur [10, 39]. In the case of the investigated anisotropic material the failure mode also depends on loading direction with more small sheared voids for loading in RD and more pronounced shear-crack mechanisms with marginal number of initial voids for loading in DD and TD.

In the case of the load ratio $F_{1} / F_{2}=0 / 1$ (Fig. 12(b)) remarkable void growth with large pores and clear dimples occur for orientation of the specimen in RD. On the other hand, for orientation in DD the voids are smaller but also lead to dimples which are smaller compared to those after loading with orientation in RD. For specimen's orientation in TD again large pores and clear dimples similar to those observed after loading with orientation in RD are revealed by SEM. It has been shown that for isotropic materials remarkable void growth is expected to occur [25] for this high stress triaxiality $\eta=1.00$ numerically predicted for this load ratio in the center of the notch (Fig. 11(b)). This damage mode also develops in the investigated anisotropic material but orientation of the specimen during the loading process has an effect on the size of the voids. They are larger for orientation in RD and TD whereas smaller pores are visible after loading with orientation in DD.

For the load ratio $F_{1} / F_{2}=1 /+1$ (Fig. 12(c)) after $F_{1}$ -loading in RD many small voids and some shear mechanisms occur leading to sheared dimples. For $F_{1}$-loading in DD only small voids can be seen in combination with microshear-cracks and sheared voids are visible. For $F_{1}$-loading in TD some voids and remarkable micro-shear-cracks develop with small sheared dimples. However, the stress triaxiality for all $F_{1}$-loading directions was nearly identical with $\eta=0.20$ in the center of the notch (Fig. 11(c)). In this case in isotropic materials the mixed failure mode with simultaneous growth of voids and formation of micro-shear-cracks is expected to occur [10, 39]. In the investigated anisotropic aluminum alloy the damage and fracture mode is also dependent on the $F_{1}$ -loading direction with more superimposed void growth for $F_{1}$-loading in $\mathrm{RD}$ and more pronounced shear-crack mechanisms for $F_{1}$-loading in DD and TD.

Figure 12(d) shows the SEM pictures for the load ratio $F_{1} / F_{2}=1 /-1$. For example, in the case of $F_{1}$-loading in RD some small voids are visible which are sheared and superimposed by micro-shear-cracks. For $F_{1}$-loading in DD nearly no voids exist and failure is caused by accumulation of micro-shear-cracks leading to the very smooth and flat failure surface. For $F_{1}$-loading in TD again only very few voids can be seen which are sheared and superimposed by micro-shear-cracks. Compared to $F_{1}$-loading in RD in the case of $F_{1}$-loading in DD and TD less voids can be seen in the SEM pictures. Based on the results in isotropic materials $[25,29]$ micro-shear-crack behavior without formation of voids is expected to occur for the numerically predicted stress triaxiality $\eta=-0.20$ in the center of the notch (Fig. 11(d)). However, in the case of the investigated anisotropic material the damage and fracture modes also depend on the $F_{1}$-loading direction. The expected mode only occurs for loading in DD and TD whereas for loading in RD additional shearing of small voids has been detected by SEM.

In summary, although no dependence of the stress triaxiality (Fig. 11) and of the macroscopic fracture lines (Fig. 10) on the specimen's orientation with respect to the axes of the principal directions of anisotropy during loading has been revealed by the experiments and the corresponding numerical simulations the SEM pictures have clearly shown the influence of the orientation of the specimen on the damage and fracture mechanisms on the micro-scale. For all investigated load ratios there is more growth and deformation of voids during loading of specimens with orientation in RD whereas smaller or no voids occur for orientation in DD. Thus, based on the SEM high stress triaxialities lead to smaller effect of the $F_{1}$ -loading direction on damage and failure mechanisms on the micro-level. In all cases predominant formation and growth of voids occurs, only the size of the voids is larger for $F_{1}$-loading in RD and TD and smaller for specimen's orientation in DD. However, for moderate and zero stress triaxialities, a remarkable influence of the orientation of the $\mathrm{H}$-specimen during loading on these microscopic processes has been revealed. For $F_{1}$-loading in RD mixed failure mechanisms with simultaneous shearing of new or pre-existing voids and formation of micro-shear-cracks can be seen and their proportion depends on the stress triaxiality (decrease in stress triaxiality leads to more micro-shear-cracks). For specimen's orientation in 
RD

(a)

$1 / 0$

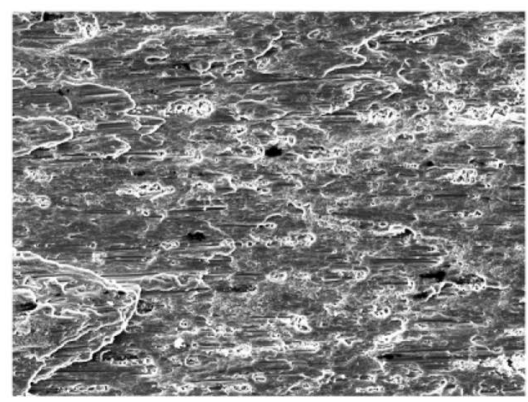

(b)

$0 / 1$

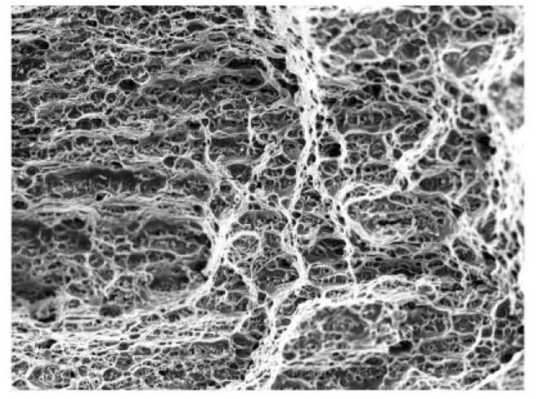

(c)

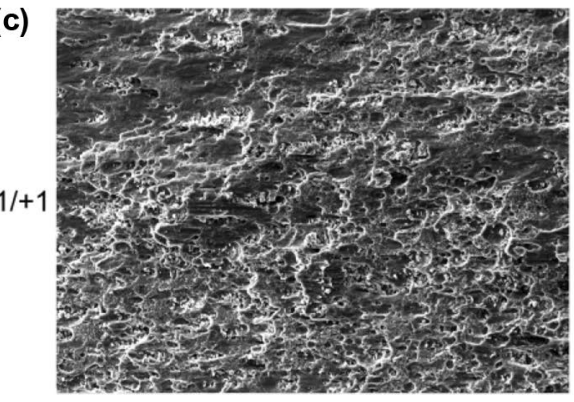

(d)

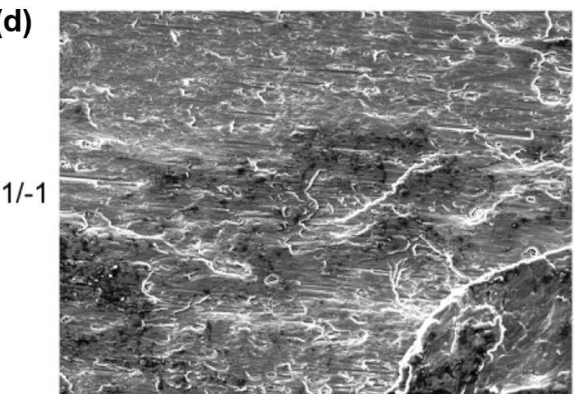

DD
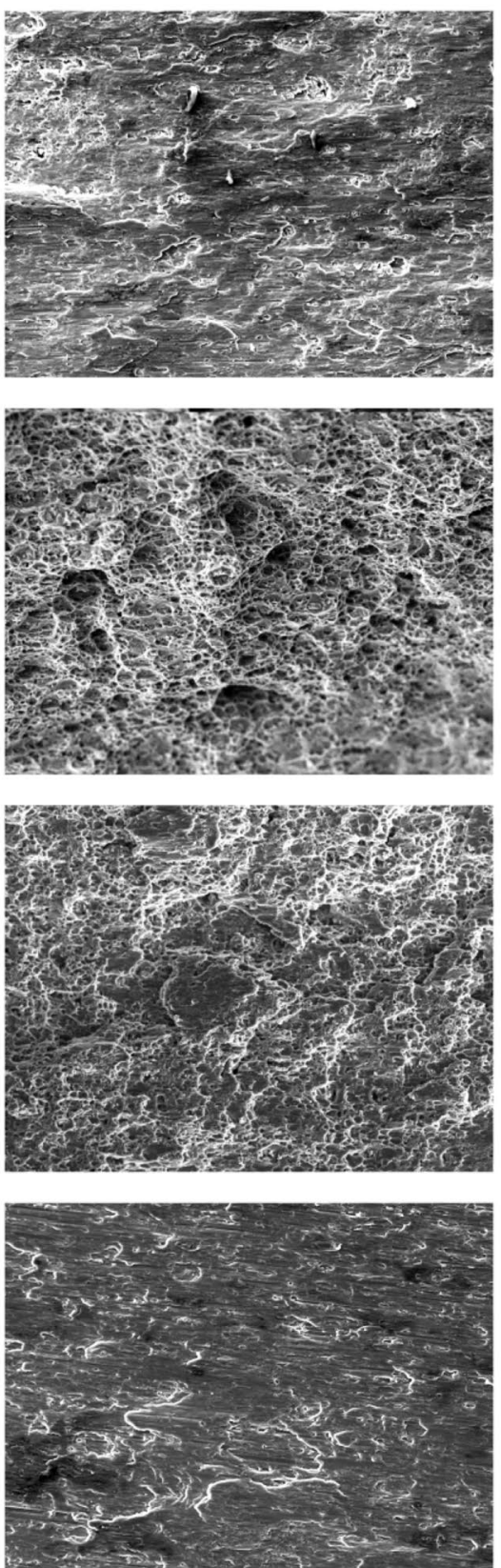

TD
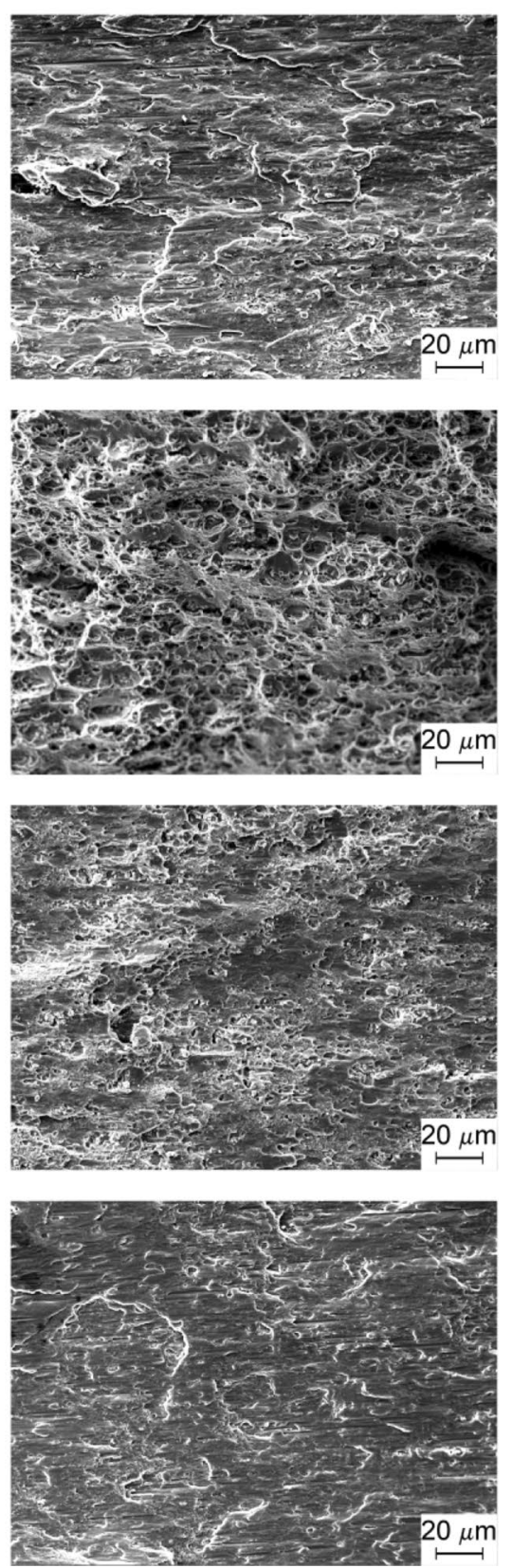

Fig. 12 Scanning electron microscopy of fracture surfaces for the load ratios (a) $F_{1} / F_{2}=1 / 0,(\mathbf{b}) F_{1} / F_{2}=0 / 1,(\mathbf{c}) F_{1} / F_{2}=1 /+1$, and (d) $F_{1} / F_{2}=1 /-1$

DD, at the same level of stress triaxiality the formation of micro-shear-cracks is more predominant and growth of voids is lower.

\section{Conclusions}

In this paper a new and innovative experimental-numerical program has been discussed to improve prediction of stressstate-dependent formation of damage and initiation of fracture in sheet metals. The effect of the loading conditions on damage and fracture behavior of the anisotropic aluminum alloy EN AW 2017A has been investigated in detail. Various experiments and numerical simulations with the biaxially loaded $\mathrm{H}$-specimen have been carried out with focus on different load ratios and loading directions with respect to the principal axes of anisotropy. These biaxial experiments allow consideration of a wide range of stress states caused by different load combinations. 
The numerical analysis is based on Hill's yield criterion. Material parameters are identified by results of uniaxial tension and shear tests. A combined method using the yield criterion and the $r$-values is proposed to accurately predict both the yield stresses and the $r$-values in specimens with different loading directions. With these parameters the biaxial experiments can be well numerically simulated and based on the numerical analysis stress triaxialities have been detected allowing interpretation of observed failure modes.

The experiments with the biaxially loaded $\mathrm{H}$-specimen have shown that the load ratio as well as the orientation of the specimen with respect to the principal axes of anisotropy affect the amount of strains as well as the localization behavior and the orientation of principal strain bands. Furthermore, damage and fracture processes on the micro-level are influenced by the load ratio and the loading direction. Loading in DD leads to more pronounced micro-shear-crack mechanisms whereas during loading in RD more sheared voids can be seen.

The experimental-numerical results discussed in the present paper reveal important information on stress-statedependent damage and fracture mechanisms in anisotropic ductile metals occurring during different biaxial loading scenarios. They will be used to enhance the continuum damage framework proposed by the authors to model damage and failure in anisotropic ductile materials. The results can also be used also by other research groups to develop and to validate sophisticated damage and fracture models to accurately predict safety and lifetime of anisotropic metals in engineering structures.

Acknowledgements This research was funded by the Deutsche Forschungsgemeinschaft (DFG, German Research Foundation) - Project number 394286626. This financial support is gratefully acknowledged.

Funding Open Access funding enabled and organized by Projekt DEAL.

\section{Declarations}

Conflict of Interest The authors declare that they have no conflict of interest.

Open Access This article is licensed under a Creative Commons Attribution 4.0 International License, which permits use, sharing, adaptation, distribution and reproduction in any medium or format, as long as you give appropriate credit to the original author(s) and the source, provide a link to the Creative Commons licence, and indicate if changes were made. The images or other third party material in this article are included in the article's Creative Commons licence, unless indicated otherwise in a credit line to the material. If material is not included in the article's Creative Commons licence and your intended use is not permitted by statutory regulation or exceeds the permitted use, you will need to obtain permission directly from the copyright holder. To view a copy of this licence, visit http://creativecommons.org/licenses/by/4.0/.

\section{References}

1. Stanić D, Zovko Brodarac Z, Li L (2020) Influence of copper addition in AlSi7 $\mathrm{MgCu}$ alloy on microstructure development and tensile strength improvement. Metals 10:1623. https://doi.org/10. 3390/met10121623

2. Yang G, Kim JK (2021) An overview of high yield strength twinninginduced plasticity steels. Metals 11:124. https://doi.org/10. 3390/met11010124

3. Myint PW, Hagihara S, Tanaka T, Taketomi S, Tadano Y (2017) Determination of the values of critical ductile fracture criteria to predict fracture initiation in punching processes. J Manuf Mater Process 1:12. https://doi.org/10.3390/jmmp1020012

4. Brünig $\mathrm{M}$ (2016) A thermodynamically consistent continuum damage model taking into account the ideas of CL Chow. Int J Damage Mech 25:1130-1141. https://doi.org/10.1177/ 1056789516639119

5. Živković J, Dunić V, Milovanović V, Pavlović A, Živković M (2021) A modified phase-field damage model for metal plasticity at finite strains: Numerical development and experimental validation. Metals 11:47. https://doi.org/10.3390/met11010047

6. Gerke S, Adulyasak P, Brünig M (2017) New biaxially loaded specimens for the analysis of damage and fracture in sheet metals. Int J Solids Struct 110-111:209-218. https://doi.org/10.1016/j. ijsolstr.2017.01.027

7. Bai Y, Wierzbicki T (2008) A new model of metal plasticity and fracture with pressure and Lode dependence. Int J Plast 24:10711096. https://doi.org/10.1016/j.ijplas.2007.09.004

8. Brünig M, Chyra O, Albrecht D, Driemeier L, Alves M (2008) A ductile damage criterion at various stress triaxialities. Int J Plast 24:1731-1755. https://doi.org/10.1016/j.ijplas.2007.12.001

9. Driemeier L, Brünig M, Micheli G, Alves M (2010) Experiments on stress-triaxiality dependence of material behavior of aluminum alloys. Mech Mater 42:207-217. https://doi.org/10.1016/j.mechmat. 2009.11.012

10. Bao Y, Wierzbicki T (2004) On fracture locus in the equivalent strain and stress triaxiality space. Int J Mech Sci 46:81-98. https:// doi.org/10.1016/j.ijmecsci.2004.02.006

11. Gao X, Zhang G, Roe C (2010) A study on the effect of the stress state on ductile fracture. Int J Damage Mech 19:75-94. https://doi. org/10.1177/1056789509101917

12. Li H, Fu MW, Lu J, Yang H (2011) Ductile fracture: Experiments and computations. Int J Plast 27:147-180. https://doi.org/ 10.1016/j.ijplas.2010.04.001

13. Dunand M, Mohr D (2011) On the predictive capabilities of the shear modified Gurson and the modified Mohr-Coulomb fracture models over a wide range of stress triaxialities and Lode angles. $\mathrm{J}$ Mech Phys Solids 59:1374-1394. https://doi.org/10.1016/j.jmps. 2011.04.006

14. Roth CC, Mohr D (2016) Ductile fracture experiments with locally proportional loading histories. Int J Plast 79:328-354. https://doi. org/10.1016/j.ijplas.2015.08.004

15. Lou Y, Chen L, Clausmeyer T, Tekkaya AE, Yoon JW (2017) Modeling of ductile fracture from shear to balanced biaxial tension for sheet metals. Int J Solids Struct 112:169-184. https://doi. org/10.1016/j.ijsolstr.2016.11.034

16. Liu Y, Kang L, Ge H (2019) Experimental and numerical study on ductile fracture of structural steels under different stress states. J Constr Steel Res 158:381-404. https://doi.org/10.1016/j.jcsr.2019. 04.001

17. Lin SB, Ding JL (1995) Experimental study of the plastic yielding of rolled sheet metals with the cruciform plate specimen. Int J Plast 11:583-604. https://doi.org/10.1016/S0749-6419(94) 00024-7 
18. Green DE, Neale KW, MacEwen SR, Makinde A, Perrin R (2004) Experimental investigation of the biaxial behaviour of an aluminum sheet. Int J Plast 20:1677-1706. https://doi.org/10.1016/j. ijplas.2003.11.012

19. Kuwabara T (2007) Advances in experiments on metal sheets and tubes in support of constitutive modeling and forming simulations. Int J Plast 23:385-419. https://doi.org/10.1016/j.ijplas.2006.06. 003

20. Kulawinski D, Nagel K, Henkel S, Hübner P, Fischer H, Kuna M, Biermann H (2011) Characterization of stress-strain behavior of a cast TRIP steel under different biaxial planar load ratios. Eng Fract Mech 78:1684-1695. https://doi.org/10.1016/j.engfracmech. 2011.02.021

21. Barroso A, Correa E, Freire J, Paris F (2018) A devise for biaxial testing in uniaxial machines design, manufacturing and experimental results using cruciform specimens of composite materials. Exp Mech 58:49-53. https://doi.org/10.1007/s11340-017-0327-6

22. Demmerle S, Boehler JP (1993) Optimal design of biaxial tensile cruciform specimens. J Mech Phys Solids 41:143-181. https://doi. org/10.1016/0022-5096(93)90067-P

23. Song X, Leotoing L, Guines D, Ragneau E (2017) Characterization of forming limits at fracture with an optimized cruciform specimen: Application to DP600 steel sheets. Int J Mech Sci 126:35-43. https://doi.org/10.1016/j.ijmecsci.2017.03.023

24. Nasdala L, Husni AH (2020) Determination of yield surfaces in accordance with ISO 16842 using an optimized cruciform test specimen. Exp Mech 60:815-832. https://doi.org/10.1007/ s11340-020-00601-9

25. Liedmann J, Gerke S, Barthold FJ, Brünig M (2020) Shape optimization of the $\mathrm{X} 0$-specimen: Theory, numerical simulation and experimental verification. Comput Mech 66:1275-1291. https:// doi.org/10.1007/s00466-020-01900-7

26. Tsutamori H, Amaishi T, Chorman RR, Eder M, Vitzthum S, Volk W (2020) Evaluation of prediction accuracy for anisotropic yield functions using cruciform hole expansion test. J Manuf Mater Process 4:43. https://doi.org/10.3390/jmmp4020043

27. Brünig M, Brenner D, Gerke S (2015) Stress state dependence of ductile damage and fracture behavior: Experiments and numerical simulations. Eng Fract Mech 141:152-169. https://doi.org/10. 1016/j.engfracmech.2015.05.022

28. Brünig M, Gerke S, Zistl M (2019) Experiments and numerical simulations with the $\mathrm{H}$-specimen on damage and fracture of ductile metals under non-proportional loading paths. Eng Fract Mech 217. https://doi.org/10.1016/j.engfracmech.2019.106531
29. Brünig M, Zistl M, Gerke S (2020) Biaxial experiments on characterization of stress-state-dependent damage in ductile metals. Prod Eng 14:87-93. https://doi.org/10.1007/s11740-019-00930-2

30. Hill R (1948) A theory of the yielding and plastic flow of anisotropic metals. Proc R Soc London 193:281-297

31. Shen F, Münstermann S, Lian J (2020) Investigation on the ductile fracture of high-strength pipeline steels using a partial anisotropic damage mechanics model. Eng Fract Mech 227. https://doi.org/ 10.1016/j.engfracmech.2020.106900

32. Marek A, Davis FM, Kim JH, Pierron F (2020) Experimental validation of the sensitivity-based virtual fields for identification of anisotropic plasticity models. Exp Mech 60:639-664. https:// doi.org/10.1007/s11340-019-00575-3

33. Barlat F, Aretz H, Yoon JW, Karabin ME, Brem JC, Dick RE (2005) Linear transformation-based anisotropic yield functions. Int J Plast 21:1009-1039. https://doi.org/10.1016/ijplas.2004.06. 004

34. Ha J, Baral M, Korkolis Y (2018) Plastic anisotropy and ductile fracture of bake-hardened AA6013 aluminum sheet. Int J Solids Struct 155:123-139. https://doi.org/10.1016/ijsolstr.2018.07.015

35. Hu Q, Yoon JW, Manopulo N, Hora P (2021) A coupled yield criterion for anisotropic hardening with analytical description under associated flow rule: Modeling and validation. Int J Plast 136. https://doi.org/10.1016/j.ijplas.2020.102882

36. Stoughton TB, Yoon JW (2009) Anisotropic hardening and nonassociated flow in proportional loading of sheet metals. Int J Plast 25:1777-1817. https://doi.org/10.1016/j.ijplas.2009.02.003

37. Spitzig WA, Richmond O (1984) The effect of pressure on the flow stress of metals. Acta Metall 32:457-463

38. Voce $\mathrm{E}$ (1955) A practical strain-hardening function. Metallurgia 51:219-226

39. Brünig M, Zistl M, Gerke S (2021) Numerical analysis of experiments on damage and fracture behavior of differently preloaded aluminum alloy specimens. Metals 11:381. https://doi.org/10. 3390/met11030381

Publisher's Note Springer Nature remains neutral with regard to jurisdictional claims in published maps and institutional affiliations. 